\title{
Clinicopathological Correlation and Varied Histomorphological Changes in Xanthogranulomatous Cholecystitis (XGC)
}

\author{
Sufian Zaheer ${ }^{1 *}$, Minakashi Chouhan ${ }^{2}$, Sachin S Kolte ${ }^{3}$ and Rashmi Arora ${ }^{3}$ \\ ${ }^{1}$ Associate Professor, Department of Pathology, Vardhman Mahavir Medical College, Safdarjung Hospital, India \\ ${ }^{2}$ Senior Resident, Department of Pathology, Vardhman Mahavir Medical College, Safdarjung Hospital, India \\ ${ }^{3}$ Professor, Department of Pathology, Vardhman Mahavir Medical College, Safdarjung Hospital, India
}

*Corresponding author: Dr. Sufian Zaheer, Associate Professor, Department of Pathology, Vardhman Mahavir Medical College, Safdarjung Hospital, $4^{\text {th }}$ Floor, College Building, New Delhi-110029, India

\begin{abstract}
Xanthogranulomatous cholecystitis (XGC) is a rare inflammatory disease of the gallbladder characterized by focal or diffuse destructive inflammation of the gall bladder comprising of mainly chronic inflammatory cells, fibrous tissue and lipid laden macrophages. The present study correlated the clinicopathological findings and studied the various histomorphological changes in Xanthogranulomatous cholecystitis (XGC). In a period of one year from January 2018 to June 2019, 22 patients were diagnosed as Xanthogranulomatous cholecystitis at our hospital. Various histomorphological changes were studied in them. The radiological findings and clinical details of the patient (age, sex, signs and symptoms) were also reviewed and analysed. The incidence of XGC was $1.8 \%$. There were 20 female patients and 02 male patient showing female preponderance for the disease. Age range was from 26 to 60 years with a mean age of 43 years. Most common presentation was pain abdomen followed by dyspepsia, nausea and vomiting and fever. In 2 patients, gall bladder carcinoma was suspected radiologically as well as clinically hence underwent extended cholecystectomy but were later diagnosed as a benign entity.

Abdominal ultrasound and CT scan showed marked thickening of the gall bladder wall in 22 cases. Gall stones were seen in 12 cases. Histologically gall bladder showed mucosal ulceration, foamy macrophages, other inflammatory cells - lymphocytes, plasma cells, eosinophils, giant cellsforeign body type and touton type, fibrosis, cholesterol clefts and bile pigment in varying proportion. In two cases, epithelial dysplasia in gallbladder lining was seen and serosa showed mild dysplastic glands. Liver tissue showed portal tract inflammation, fatty changes in parenchyma, bile duct proliferation and inflammation in surrounding fat and connective tissue.
\end{abstract}

Although radiology is helpful in detecting the abnormalities of gall bladder, but many times fail to differentiate between gallbladder malignancy and XGC. Definite diagnosis can be made only on histopathology. Ducts of luschka found in serosa can mimic malignancy when they show reactive atypia. Carcinoma of gallbladder is more frequently associated with XGC than other forms of cholecystitis. Therefore this condition needs to be diagnosed carefully because of its confusion with malignancy.

\section{Introduction}

Xanthogranulomatous cholecystitis (XGC) is a benign, rare variant of chronic cholecystitis characterized by focal or diffuse inflammation of gall bladder. Its incidence is variable in different studies ranging from $1.2 \%$ to $8.9 \%$. It is very often misdiagnosed as gall bladder cancer often on clinical and radiological findings, and sometimes even on histopathological evaluation. XGC was first described by Christensen and Ishak [1], as Fibroxanthogranulomatous inflammation in the year 1970. Later the condition was described as ceroid like histiocytic granuloma of gall bladder (GB) and biliary granulomatous cholecystitis [2,3]. It is characterized by focal or diffuse destructive inflammatory process comprising of mainly chronic inflammatory cells, fibrous tissue and lipid laden macrophages. Grossly it shows yellow brown nodules with abnormal and asymmetrical thickening of the gall bladder wall. Its pathogenesis is not clear. It is thought to start as a biliary obstruction with acute or chronic cholelithiasis and increasing intra-gallbladder pressure. This pressure causes rupture

Citation: Zaheer S, Chouhan M, Kolte SS, Arora R (2020) Clinicopathological Correlation and Varied Histomorphological Changes in Xanthogranulomatous Cholecystitis (XGC). Int J Pathol Clin Res 6:107. doi.org/10.23937/2469-5807/1510107

Accepted: April 21, 2020: Published: April 23, 2020

Copyright: (C) 2020 Zaheer S, et al. This is an open-access article distributed under the terms of the Creative Commons Attribution License, which permits unrestricted use, distribution, and reproduction in any medium, provided the original author and source are credited. 
of Rokitansky-Aschoff (RA) sinuses or mucosal ulcer with release of bile in interstitial tissue and a consequent Xanthogranulomatous inflammatory reaction [3]. The inflammatory process is often extensive and may extend to adjacent organs forming dense adhesions and large mass of inflammatory tissue surrounding the gall bladder.

\section{Objectives}

The study aimed to determine clinicopathological correlation and various histomorphological changes in Xanthogranulomatous cholecystitis (XGC).

\section{Material and Methods}

The study was done on prospectively collected data of patient operated upon in our department with a preoperative diagnosis of cholecystitis from January 2018 to June 2019. All patients who underwent radical cholecystectomy were considered for study. A total of 1250 patients underwent cholecystectomy during this period. Among these, 22 patients were diagnosed as Xanthogranulomatous cholecystitis. We studied various histomorphological changes in these cases. Particular stress was given on mucosal ulceration, wall thickness, fibrosis, evidence of epithelial dysplasia and serosal changes. We also reviewed the radiological findings and clinical details of the patient including age, sex, signs and symptoms.

All the gallbladder specimens received in our department were formalin fixed. They were opened longitudinally and sections were taken from neck, body and fundus region which were stained with $\mathrm{H} \& \mathrm{E}$ stain and then examined under the microscope.

\section{Results}

The study was done on prospectively collected data of patients operated upon in our department with a preoperative diagnosis of cholecystitis / carcinoma gall bladder from January 2018 to June 2019.

Out of 1250 cases, 22 case were diagnosed as Xanthogranulomatous cholecystitis, giving an annual incidence of $1.8 \%$. There were 20 female patients and 02 male patient showing female preponderance for the disease. Age of the patients ranged from 26 to 60 years with a mean age of 43 years. Most of the patients presented with pain abdomen (18), nausea and vomiting (9), anorexia and weight loss (10), dyspepsia (16) and fever (8).

USG and/or CECT abdomen showed focal or diffuse wall thickening in body and fundus of the gallbladder (Figure 1) in all the 22 patients, continuous mucosal line in 12 and submucosal hypoattenuating nodule in 6 patients. Cholelithiasis was seen in 12 patients and choledocholithiasis in 1 patient only.

\section{Macroscopic and histopathological findings}

Open cholecystectomy was done in most of the patients while 2 of them underwent extended cholecystectomy with right sub-hepatic drain placement under GA and epidural anesthesia. In these two cases, GB was densely adherent with omentum, duodenum, liver and transverse colon intraoperatively. When we received them in our department, they were surrounded by attached structures including liver, omentum, cystic duct and duodenum. Table 1 gives the macroscopic and microscopic findings in all 22 patients. The gall bladder showed wall thickness ranging from 0.7 to $1.5 \mathrm{~cm}$ along with presence of small yellow brown nodules (Figure 2). A definite growth was identified in one case while another case showed papillary excrescences. Both these cases were clinically suspected as malignancy so underwent extended cholecystectomy. Cholesterol or pig-

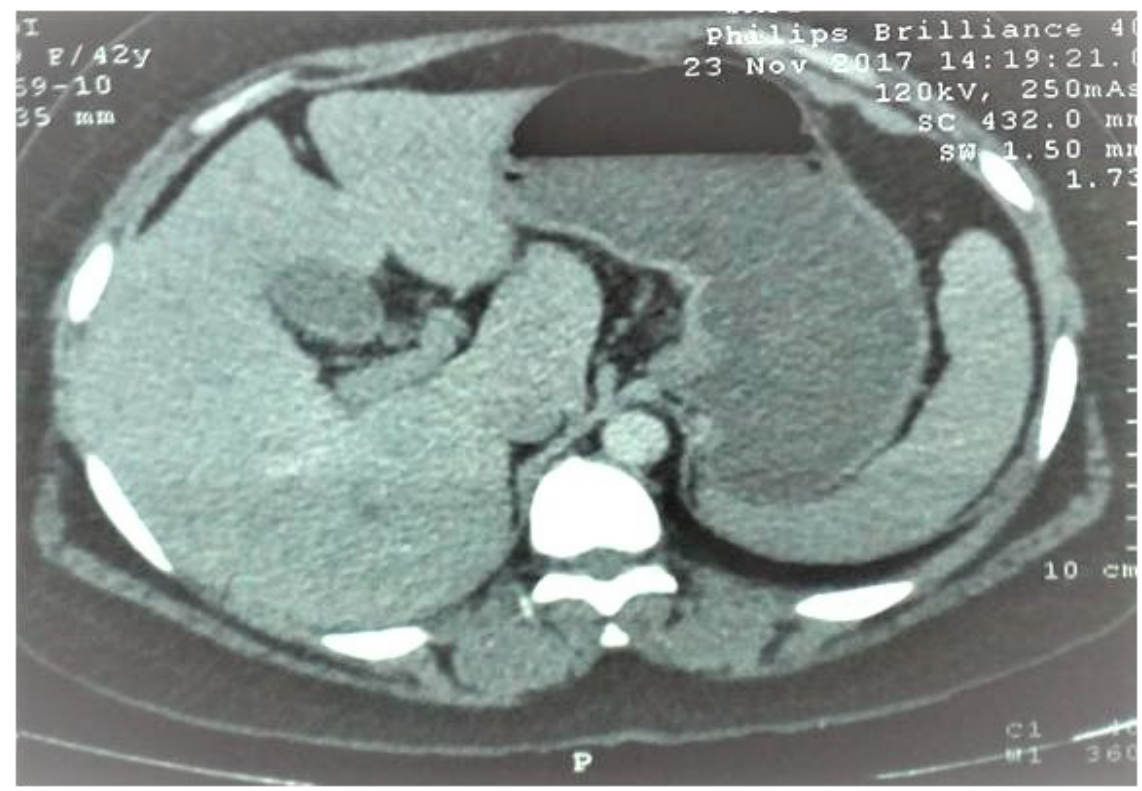

Figure 1: CECT abdomen showing thickening of gallbladder wall. 
Table 1: Macroscopic and histomorphological features in Xanthogranulomatous cholecystitis.

\begin{tabular}{|l|l|}
\hline A. Macroscopic findings & N \\
\hline 1. Thickened wall & 22 \\
2. Yellow brown nodules & 08 \\
3. Papillary excrescences & 01 \\
4. Definite growth & 01 \\
5. Gall stones & 12 \\
6. Gallbladder adhered to liver/omentum/duodenum & 02 \\
\hline
\end{tabular}

\section{B. Microscopic findings}

\begin{tabular}{l|l} 
1. Mucosal ulceration & 11 \\
2. Foamy macrophages: & 16 \\
Sheets & 06
\end{tabular}

\section{Scattered}

3. Other inflammatory cells:

Lymphocytes

\section{2}

08

01

01

12

02

Plasma cells

4. Giant cells:

\begin{tabular}{l|l} 
Foreign body type & 06
\end{tabular}

\begin{tabular}{|l|l|}
\hline Touton type & 13
\end{tabular}

\begin{tabular}{l|l} 
5. Fibrosis & 20
\end{tabular}

\begin{tabular}{|l|l}
\hline 6. Epithelial dysplasia in gallbladder lining & 02
\end{tabular}

\begin{tabular}{l|l} 
7. Serosa showing mild dysplastic glands & 01
\end{tabular}

\begin{tabular}{l|l} 
8. Cholesterol clefts & 10
\end{tabular}

\begin{tabular}{l|l} 
9. Bile pigment & 14
\end{tabular}

10. Liver:

\begin{tabular}{l|l} 
Portal tract inflammation & 03
\end{tabular}

\begin{tabular}{|l|l}
\hline Fatty changes in parenchyma & 02
\end{tabular}

\begin{tabular}{l|l}
\hline Bile duct proliferation & 03
\end{tabular}

\begin{tabular}{|l|l} 
11. Inflammation in surrounding fat and connective tissue & 02
\end{tabular}

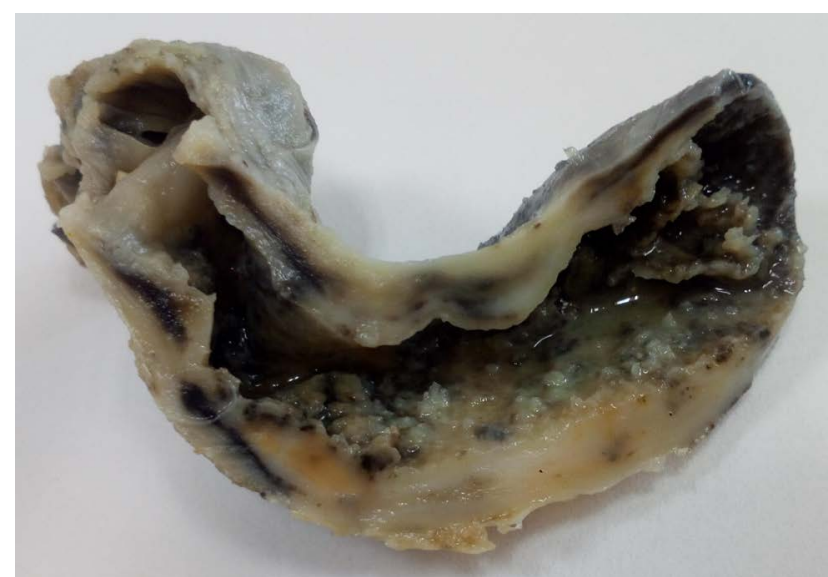

Figure 2: Gallbladder wall thickening with yellowish areas.

ment stones were identified in 12 patients.

Histologically, the GB wall was markedly thickened showing severe inflammation and fibrosis (Figure 3 ). Mucosal ulceration was also seen in 11 cases (Figure 4). Foamy macrophages were seen arranged in sheets and scattered singly along with chronic inflammatory cells comprising of lymphocytes, plasma cells and few eosinophils. Foamy macrophages were large round to

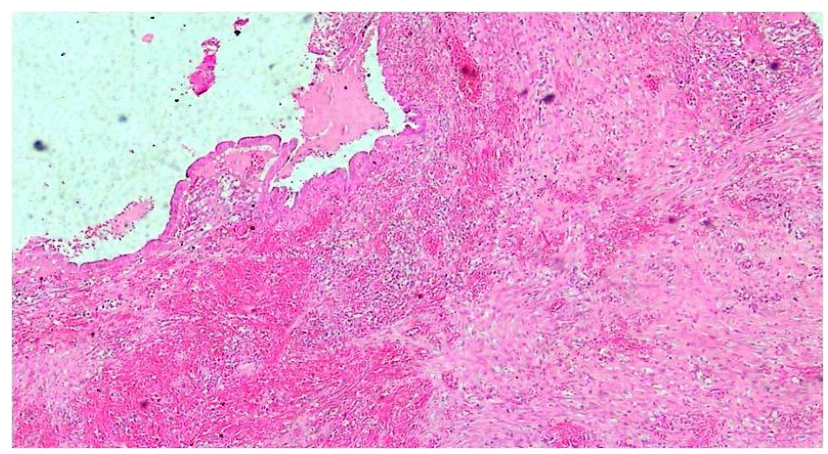

Figure 3: Gallbladder wall showing chronic inflammation, thickening and fibrosis (H\&E $4 \mathrm{x})$.

oval cells with abundant foamy to clear lipid containing cytoplasm with single nucleus (Figure 5). Bile pigment, cholesterol clefts (Figure 6) and multinucleated giant cells (Figure 7) were also identified. Two types of giant cells were seen - foreign body giant cell with haphazard arrangement of nucleus and Touton giant cells with a central ring of nuclei with the peripheral clear cytoplasm due to accumulation of lipid. Lymphocytic infiltration was present in all the cases while eosinophils were seen in fewer cases only. Gall bladder wall was showing fibrosis which was extending to liver. In one case with 


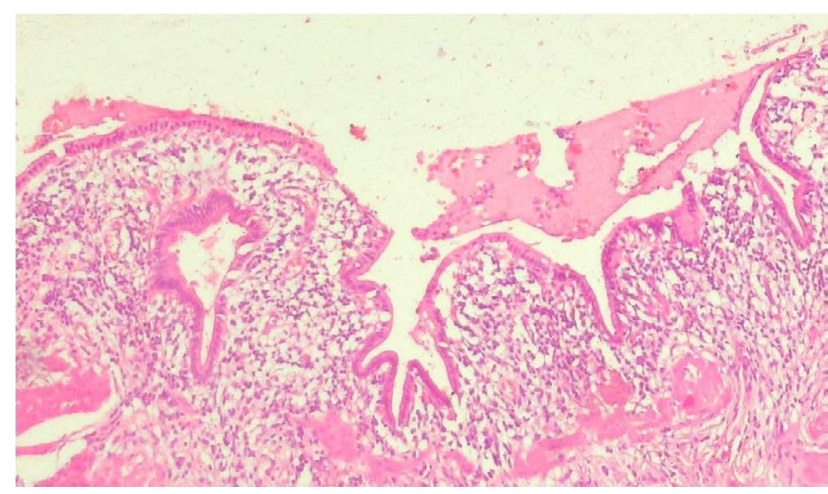

Figure 4: Chronic inflammation including macrophages and focal mucosal ulceration in the gall bladder mucosa. (H\&E 10x).

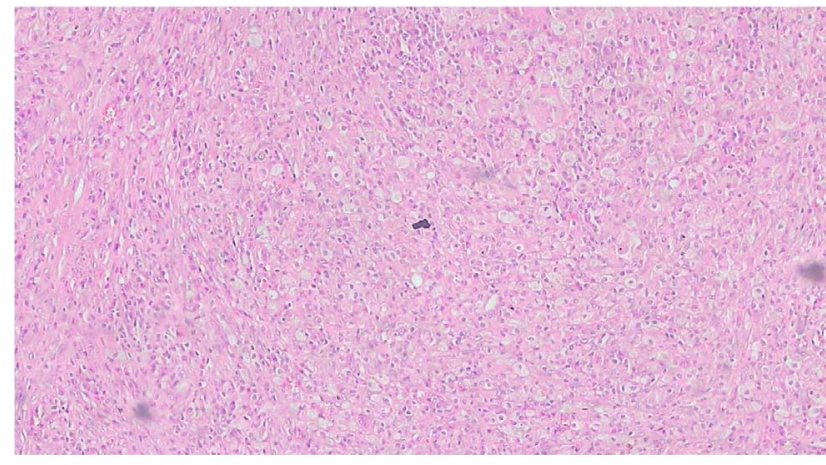

Figure 5: Gallbladder wall showing foamy macrophages in sheets (H\&E 10x).

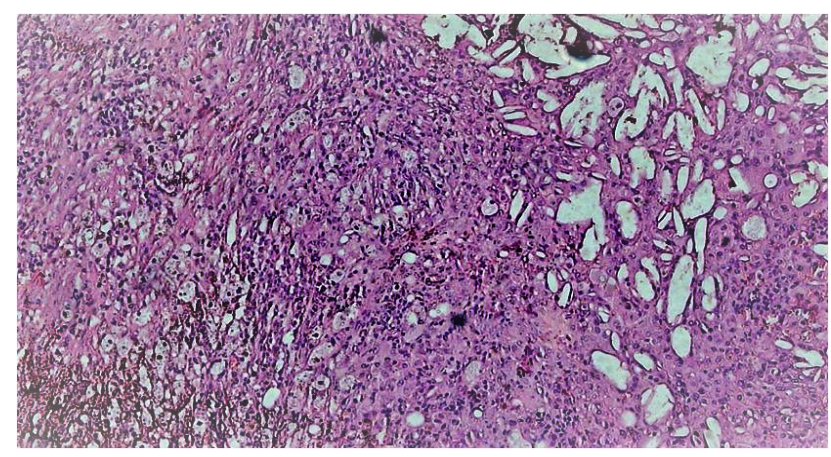

Figure 6: Foamy macrophages and cholesterol clefts in gallbladder wall.

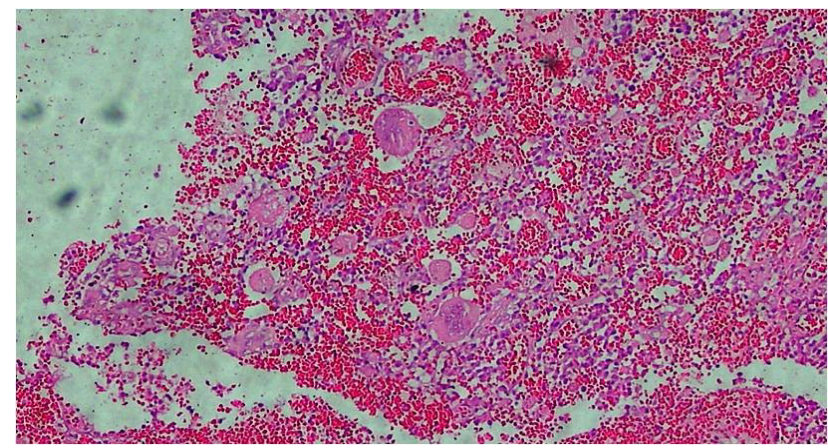

Figure 7: Gallbladder wall showing multinucleated giant cells.

definite growth, the gall bladder wall was markedly thickened with severe inflammation, fibrosis and foamy

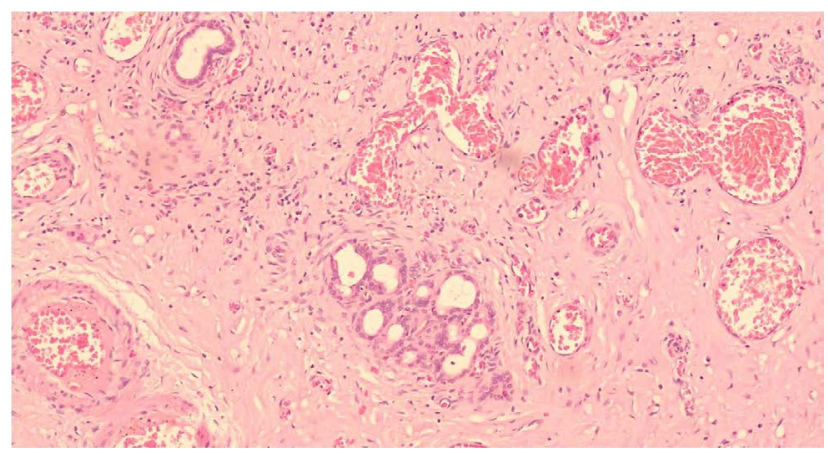

Figure 8: Proliferating ducts of luschka in gallbladder serosa.

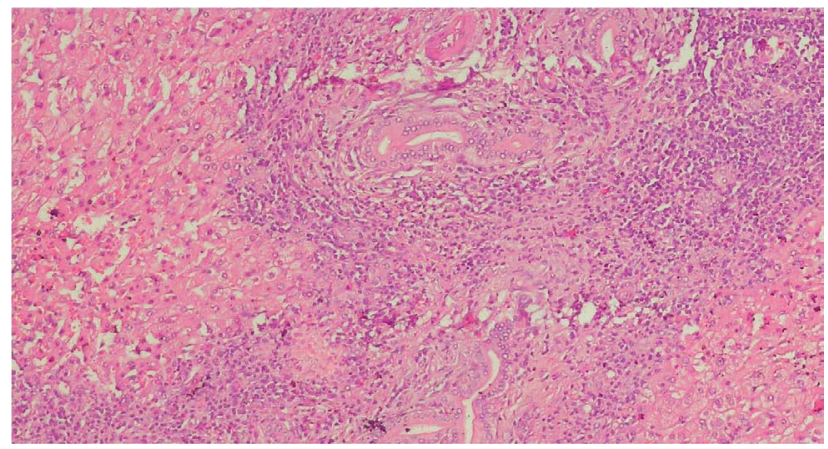

Figure 9: Inflammation and bile duct proliferation in liver bed tissue.

histiocytes which were arranged in sheets. The lining epithelium was showing mild dysplasia and few of the glands were reaching up to serosa where ducts of Luschka also showed proliferation (Figure 8). These ducts are developmental anomalies and seen in only $10 \%$ of cholecystectomies. These ducts were showing lobular aggregates of small sized ductulus which were lined by bland looking cuboidal to columnar type epithelium associated with centrally located larger ductulus surrounded by concentric fibrosis. There was marked inflammation and collection of macrophages surrounding the gallbladder extending into surrounding fat and connective tissue. The case showing papillary excrescences showed mucosal ulceration and gall bladder wall thickening due to chronic inflammatory cells, fibrosis and foamy histiocytes.

Liver tissue showed chronic inflammation in portal tract and focal fatty change in liver parenchyma. There was proliferation of bile ducts within liver tissue surrounded by fibrosis and chronic inflammatory infiltrate (Figure 9).

\section{Discussion}

XGC is a rare variant of cholecystitis. Incidence of Xanthogranulomatous cholecystitis was $1.8 \%$ in our study which correlates with that of the study conducted by KM Roberts and MA parson [4] with incidence of $1.8 \%$. Incidence reported by Takashahi, et al. [3] was 1.2\%, Guzman-Valdivia G [5] in Mexico was $1.46 \%$, by Karbulut Z [6] was $1.5 \%$. However there are studies which show higher 
incidence of $8.9 \%$ by Dixit VK [7] in Varanasi, India and $10 \%$ by Krishna RP, et al. [8] in Lucknow, India.

There were 20 females and 02 male patient of XGC in our study showing a female preponderance for the disease. It reveals the increased incidence of gall bladder infections in women. KM Roberts also reported the female preponderance for the disease [4].

Although radiology is helpful in detecting the abnormalities of gall bladder, but many times fail to differentiate between gallbladder malignancy and XGC. Radiological changes seen in XGC are abnormal thickening of the wall, severe proliferative fibrosis along with scarring, formation of multiple yellow-brown intramural nodules and extensive involvement of adjacent organs. These changes may mimic malignancy [9]. Some studies tried to differentiate these two conditions. Uchiyama, et al. [10], emphasized that enhanced continuous mucosal line in CT image with gallstones was highly suggestive of XGC. Gall bladder wall thickening was most common finding on USG in our study. Lichtman JB and Parra JA [11] reported moderate to marked thickening of Gall bladder wall with hypoechoic nodules. Kimm PN [12] reported intramural nodules in GB in $72 \%$ cases of XGC. There is frequent association of gall stones with XGC. In our study gall stones were seen in $54 \%$ of patients. Similar findings were reported by Hideki Suzuki [9]. XGC can present with complications including GB perforation, abscess formation, duodenal fistula, and extension of the inflammatory process to adjacent abdominal organs, such as the liver and transverse colon [13]. Our study also reported a complication where inflammation was extended to the adjacent organs leading to extended cholecystectomy.

In our study, we have observed various histopathological changes of XGC in gall bladder, liver parenchyma, portal tracts, bile ducts and adjacent fat and connective tissue. Many times it is difficult to differentiate XGC from malignancy, especially those cases with involvement of surrounding structures. A study by KM Roberts [6] reported various histomorphological changes in XGC. They described two types of xanthoma cells - round cells and spindle cells with elongated nuclei. These spindle shaped cells were arranged in the form of storiform pattern. These spindle shaped cells raise the suspicion of malignancy. They also described the presence of Cholesterol clefts, lipid droplets, hemosiderin deposits, giant cells of foreign body and Touton type and extravasated bile in their study of 13 cases. In our study we reported histopathological changes in liver including focal fatty changes in its parenchyma, bile ducts proliferation and portal tract inflammation. Hideki Suzuki [9] reported the presence of hyperplasia in the gallbladder mucosa, with the absence of atypical changes or malignant cells. We too reported the presence of mild dysplasia in mucosal lining of gall bladder in our study. Such dysplastic glands were reaching up to the serosa but there were no other features of malignancy including cellular atypia, pleomorphism and increased atypical mitotic figures. There were some ducts in the serosa which exist singly or a network of ducts which communicate with intrahepatic bile ducts and gallbladder. These ducts are known as ducts of Luschka. They are developmental anomalies and seen in $10 \%$ of cholecystectomies only. When these ducts are numerous and in the background of inflammatory infiltrate and show reactive atypia, they are mistaken for invasive adenocarcinoma [14]. Rajab $R$ in their study described the features of ducts of Luschka and how to differentiate it from malignancy. Ducts of Luschka are located in adventitia only while the malignant glands are seen throughout the full thickness of Gallbladder. Architecture is haphazard in malignancy while it is lobular or linear in ducts of Luschka. Fibrosis is concentric in ducts of Lushka and irregular in malignancy. Ducts of lushka show reactive features only while malignancy show nuclear variation (4:1) within a single gland. Malignancy show frequent mitosis, vascular and peri-neural invasion, all these features are absent in ducts of Lushka. Carcinoma of gallbladder is more frequently associated with XGC than other forms of cholecystitis [15]. Therefore this condition needs to be diagnosed carefully because of its confusion with malignancy.

\section{Conflict of Interest}

Nil.

\section{Statement of Equal Authors' Contribution}

All authors have equally contributed for the manuscript.

\section{References}

1. Christensen $A H$, Ishak KG (1970) Benign tumors and pseudotumours of the gall bladder. Report of 180 cases. Arch Pathol 90: 423-432.

2. Amazon K, Rywlin AM (1980) Ceroid granulomas of the gall bladder. Am J Clin Pathol 73: 123-127.

3. Takahashi K, Oka K, Hakozaki H, Kojima M (1976) Ceroidlike histiocytic granuloma of gall bladder --a previously undescribed lesion. Acta Pathol Jpn 26: 25-46.

4. Roberts KM, Parsons MA (1987) Xanthogranulomatous cholecystitis: Clinicopathological study of 13 cases. J Clin Pathol 40: 412-417.

5. Guzmán-Valdivia G (2005) Xanthogranulomatous cholecystitis in laparoscopic surgery. J Gastrointest Surg 9: 494497.

6. Karabulut Z, Besim H, Hamamci O, Bostanoğlu S, Korkmaz A (2003) Xanthogranulomatous cholecystitis. Retrospective analysis of 12 cases. Acta Chir Belg 103: 297-299.

7. Dixit VK, Prakash A, Gupta A, Pandey M, Gautam A, et al. (1998) Xanthogranulomatous cholecystitis. Dig Dis Sci 43: 940-942.

8. Krishna RP, Kumar A, Singh RK, Sikora S, Saxena R, et al. (2008) Xanthogranulomatous inflammatory strictures of extrahepatic biliary tract: Presentation and surgical management. J Gastrointest Surg 12: 836-841. 
9. Hideki Suzuki, Satoshi Wada, Kenichiro Araki, Norio Kubo, Akira Watanabe, et al. (2015) Xanthogranulomatous cholecystitis: Difficulty in differentiating from gallbladder cancer. World J Gastroenterol 21: 10166-10173.

10. Uchiyama K, Ozawa S, Ueno M, Hayami S, Hirono S, et al. (2009) Xanthogranulomatous cholecystitis: The use of preoperative CT findings to differentiate it from gallbladder carcinoma. J Hepatobiliary Pancreat Surg 16: 333-338.

11. Lichtman JB, Varma VA (1987) Ultrasound demonstration of xanthogranulomatous cholecystitis. J Clin Ultrasound 15: 342-345.

12. Kim PN, Lee SH, Gong GY, Kim JG, Ha HK, et al. (1999) Xanthogranulomatous cholecystitis: Radiologic findings with histologic correlation that focuses on intramural nodules. AJR Am J Roentgenol 172: 949-953.

13. Parsons MA, Harris SC, Grainger RG, Ross B, Smith JA (1986) Fistula and sinus formation in xanthogranulomatous cholecystitis. A clinicopathological review and report of four cases. Br J Urol 58: 488-493.

14. Rajab R, Meara N, Chang F (2007) Florid ducts of Luschka mimicking a well differentiated adenocarcinoma of the gallbladder. Int J pathol 6: 1-5.

15. Benbow EW (1989) Xanthogranulomatous cholecystitis associated with carcinoma of the gallbladder. Postgrad Med J 65: 528-531. 